\title{
Hybrid Deduction-Refutation Systems for FDE-Based Logics
}

\author{
Eoin Moore \\ City University of New York, The Graduate Center \\ emoore@gradcenter.cuny.edu
}

\begin{abstract}
Hybrid deduction-refutation systems are presented for four first degree entailment based logics. The hybrid systems are shown to deductively and refutationally sound and complete with respect to their logics. The proofs of completeness are presented in a uniform way. This paper builds on work in [7], where Goranko presented a deductively and refutationally sound and complete hybrid system for classical logic.
\end{abstract}

\section{Introduction}

Traditional deductive systems employ rules and axioms to generate all the validities of a logic. One may invert the question and ask for a logical calculus which computes not the validities, but rather the nonvalidities of the logic. Such systems are called refutation systems. In [7], Goranko combined the machinery of deductive systems and refutation systems to create so-called hybrid deduction-refutation systems, also called hybrid deductive systems or hybrid systems for short. Applying his system to classical logic, he created a logical calculus that is able to syntactically deduce all the validities and refute all the nonvalidities of classical logic, using only a few simple rules and axioms. His system is deductively and refutationally sound and complete for classical logic, (also called Ł-sound and complete, after Łukasiewicz,) which intuitively means that it deduces all and only the valid entailments, and refutes all and only the nonvalid entailments.

In this paper, we extend this approach to the case of first degree entailment and three extensions of first degree entailment - the logic of paradox, Kleene's strong three-valued logic, and classical logic. We present an Ł-sound and complete hybrid deductive system for each of the four logics. We prove $\mathrm{E}$-soundness and completeness for all four logics in one uniform approach. Our proof for classical logic differs from Goranko's in a way to be discussed later.

In Section 2 we outline the historical development of refutation systems and hybrid deduction-refutation systems. In Section 3 we review first degree entailment, and present some basic facts about the four logics in question. In 
Section 4 we provide definitions and results related to hybrid systems, mostly coming from Goranko's paper [7]. Our contribution comes in Section 5, where we present hybrid systems for the four FDE based logics and prove E-soundness and completeness for each. We end with some thoughts and conclusions in Section 6 .

\section{Hybrid deductive systems}

\subsection{Overview}

Consider a logical system L, with corresponding deductive system $\vdash_{\mathrm{L}}$ and semantics $\left.\right|_{\mathrm{L}}$. If our logic is sound and complete (in the traditional sense, hereafter called deductively sound and complete, ) then $\Gamma \vdash_{\mathrm{L}} \phi \Leftrightarrow \Gamma \models_{\mathrm{L}} \phi$. The completeness theorem in particular means that by only using axioms and rules of $\vdash_{\mathrm{L}}$, one may derive all valid formulas.

The idea of having a syllogism to compute all nonvalidities of a logic goes back to Aristotle. He realized that one did not need to produce a counterexample to show that a given expression was not valid. Instead, nonvalidity could be derived in a procedural way through its own system of rules.

Following Aristotle the subject was largely ignored, until it was revived by Łukasiewicz and his followers. In [11], Łukasiewicz considered a logic which treated acceptance and rejection on par. He showed Aristotle's system was refutationally incomplete for syllogisms, in that it did not reject every nonvalid syllogism. He created a system which did reject all nonvalid syllogisms of Aristotle's logic. Łukasiewicz' system itself was refutationally incomplete, though, in that there were meaningful non-syllogistic expressions in the language which were neither accepted nor rejected. His student Słupecki added two rules to the system to make it refutationally complete.

Łukasiewicz' system was perhaps the first refutation system in modern logic. A refutation system is a deductive calculus where rules are used to refute, or reject, expressions in the language. To refute here means to assert the nonvalidity of an expression. Denoting refutation with the symbol $\dashv$, refutation systems work analogously to traditional deductive calculi. If $\dashv$ is refutationally sound with respect to a logic $\models_{L}$, then $\Gamma \dashv \phi \Rightarrow \Gamma \not \nvdash_{L} \phi$. If $\dashv$ is refutationally complete with respect to $\models_{L}$, then $\Gamma \nvdash_{L} \phi \Rightarrow \Gamma \dashv \phi$. Łukasiewicz' and Słupecki therefore created a system which is refutationally sound and complete for Aristotle's logic.

Eukasiewicz also created a system that was both refutationally sound and complete, and deductively sound and complete for classical logic. As mentioned in the introduction, such systems are now called Ł-sound and complete. Denote classical logic with semantics $\models_{\mathrm{CL}}$ along with some sound and complete deductive system for it $\vdash_{\mathrm{CL}}$. Denote Lukasiewicz' system with the two operators $\vdash, \dashv$. Here is his system.

$$
\Gamma \vdash \phi \text { iff } \Gamma \vdash_{\mathrm{CL}} \phi
$$

Australasian Journal of Logic (18:6) 2021, Article no. 1 


$$
\frac{\dashv \sigma(\phi)}{\dashv \phi} \text { (reverse substitution) }
$$

where $\sigma$ is any uniform substitution, and

$$
\frac{\vdash \phi \rightarrow \psi \quad \dashv \psi}{\dashv \phi} \text { (Modus Tollens). }
$$

Since then, the study of refutation systems has grown tremendously. Refutation systems have been developed for a number of systems, including intuitionistic logic [15], intermediate logics [17], as well as major modal logics [8].

Refutation systems may be viewed as pure or hybrid. Pure refutation systems employ only the notion of $\dashv$ in their rules. They are concerned exclusively with deriving nonvalidities. Hybrid refutation deduction systems use both $\vdash$ and $\dashv$, and seek to derive the validities and nonvalidities of a logic. Łukasiewicz' system for classical logic was a hybrid system. Since then, however, much of the research in the literature has focused on pure refutation systems.

One advantage a hybrid system may enjoy over its competitors is that by using purely syntactic means one may deduce the validities and nonvalidities of the logic. One does not have to appeal to model theory once the system is proven E-sound and complete. In particular, one does not need to produce a countermodel to determine that a sentence is not valid, as one may syntactically derive that fact. From an aesthetic perspective, a hybrid deduction system also enjoys a certain elegance with it's symmetric treatment of $\vdash$ and $\dashv$, coinciding with the equal status of accept and reject in the philosophical underpinnings of refutation calculi.

Even among hybrid systems, $\vdash, \dashv$ are often treated separately, that is, they do not interact. Moreover, in establishing the rules and axioms for $\dashv$, one often has an underlying logical system in mind. (Consider the trivial refutation calculus for classical logic with axiom schema $\Gamma \dashv \phi$ iff $\Gamma \neq_{\mathrm{CL}} \phi$.) In such systems $\vdash$ can, in a sense, be seen as privileged over $\dashv$. Yet, there remains a

desire to see accept and reject, as much as is possible, as equal and interacting players. Therefore, there is motivation to produce a calculus which treats them as such.

In [7], Goranko explores the use of interacting $\vdash, \dashv$ operators in such a way. Treating the two operators on par, he creates a logical system which is E-sound and complete for classical logic, which does not apriori reference classical logic, as in the trivial example above.

Though Goranko's system is for classical logic, the framework he puts forward is fairly general, and can be applied to a wide class of logics. He lays out a procedure to create a hybrid deductive-refutation system from an underlying purely deductive system. In this paper, we extend the techniques in that paper to a family of logics based on first degree entailment.

\subsection{Other studies of hybrid refutation-deduction systems}

Previous works that dealt with rejection and assertion on par include the following, presented in chronological order. 
López-Escobar in [12] consider a Brower-Heyting-Kolmogorov style semantics for a propositional calculus, where $\neg A$ is claimed by demonstrating a refutation of $A$, with refutation being a primitive concept on par with proof. Elementary number theory is then studied in this context, with the underlying logic being the one just put forth.

In [2], Bonatti and Varzi define the notion of complimentary systems, which are proof systems where that which is derivable in one system is exactly that which cannot be derived in the other.

In [18], Tamminga and Tanaka develop the analogy between accept/reject in Łukasiewicz' systems, and true/false in first degree entailment. They create a natural deduction system for FDE with mixed use of $\vdash$ and $\dashv$ operators, where $\vdash \phi$ is interpreted as $\phi$ takes true as a truth value, and $\dashv \phi$ is interpreted as $\phi$ takes false as a truth value. This system, interesting in its own right, may be seen as a precursor of ideas in this paper and in [7]. However, it does not syntactically prove nonvalidity of entailment relations. That is, $\vdash$ is not extended to a relation between sets of formulas and formulas so that $\Gamma \dashv \phi \Leftrightarrow \Gamma \not \models \phi$, and thus the natural deduction system here would not be considered a hybrid system in the sense used in this paper.

In [16], Skura considers a logic being essentially characterized by a positive part (valid sentences) and a negative part (nonvalid sentences,) with rules and closure conditions for generating the respective parts.

In [4], Cafrera and Peltier consider a wider notion of entailment than the usual true to true. In terms of assertion and rejection, they analyse the four possible types of entailment that can be described - asserted to asserted, asserted to rejected, rejected to rejected, and rejected to asserted. They then apply their results to practical applications, for example, use in theorem provers.

Brady in [3] puts forth a relevant logic deductive system $\mathrm{L}_{1}$, and develops for it a Hilbert-style hybrid system called $L_{1_{r}}$, with interacting $\vdash_{\mathrm{L}_{1_{r}}}$ and $\dashv_{\mathrm{L}_{1_{\mathrm{r}}}}$. Among other things, $\mathrm{L}_{1_{r}}$ proves all the theorems of $\mathrm{E}_{\mathrm{FDE}}$ and rejects all the non theorems of $\mathrm{E}_{\mathrm{FDE}}$. To be more precise, let $\alpha$ be a first degree entailment, that is $\alpha$ is $\beta \rightarrow \gamma$, where $\beta$ and $\gamma$ do not contain the symbol $\rightarrow$. Then $\vdash_{\mathrm{L}_{1_{\mathrm{r}}}} \alpha$ iff $\vdash_{\mathrm{E}_{\mathrm{FDE}}} \alpha$, and $\dashv_{\mathrm{L}_{1 \mathrm{r}}} \alpha$ iff $\nvdash_{\mathrm{E}_{\mathrm{FDE}}} \alpha$. Since $\mathrm{E}_{\mathrm{FDE}}$ theorems correspond to FDE entailments, Brady's system can be seen as a precursor to the hybrid system for FDE developed in this paper. Brady's system can also be seen as an extension of our system, as $\mathrm{L}_{1_{r}}$ additionally considers formulas which are not first degree entailments. The proof technique used by Brady is rather different than the one used here, though. He uses the concept of metavaluations to prove his results. Roughly speaking, a metavaluation is a kind of truth-functional valuation of formulas where the inductive clause defining the truth value of a formula may depend also on the provability of some of its subformulas.

One important conceptual difference between Brady's system and Goranko's hybrid systems, however, is that in Goranko, $\vdash$ and $\dashv$ are used, respectively, to represent valid and nonvalid entailments, whereas in Brady, $\vdash$ and $\dashv$ are used to represent the less general concept of theorems and nontheorems. That is, Brady establishes $\dashv_{L_{1 \mathrm{r}}} \alpha$ iff $\nvdash_{\mathrm{L}_{1}} \alpha$, but it is not established that $\Gamma \dashv_{\mathrm{L}_{1 \mathrm{r}}} \alpha$ iff $\Gamma \nvdash_{L_{1}} \alpha$. We suspect the difference between proving and refuting entailments 
versus sentences in a hybrid system is closely related to the usage of sequents or a Hilbert-style formulation in the underlying calculus.

In [19], Wansing studies the relations between the speech acts of assertion and denial, and the corresponding inferential claims of proof and disproof, as well as their duals. He develops a semantics for bi-intuitionistic logic using proof, disproof, and their duals as primitives.

Goré and Postniece in [10] fruitfully employ a hybrid refutation-derivation system to create, for the first time, a cut-free sequent calculus for bi-intuitionistic logic.

Citkin in [5] creates a metalogical system to uniformly handle assertions and rejections, as well as multiple conclusion rules.

For more sources and discussion of hybrid systems, see [7]. For a history of refutation systems, see [9] or [5].

\section{Four FDE based logics}

First degree entailment (FDE) is a logical system first put forward by Belnap in [1] and expounded by Bellnap, Dunn, and others in a number of publications, for example, [6]. It can be considered as a four-valued logic, with truth values true, false, both, neither, written, respectively, as $t, f, b, n$. Designated truth values are $t$ and $b$. One of the goals of FDE was to develop a reasoning system that does not fail under the existence of contradiction. The example given in [1] is that an inconsistency in baseball World Series data should not lead a computer to mishandle flight information. Yet a computer obeying classical logic would conclude anything and everything about flight information in the presence of one inconsistent datum. To this end, the authors develop a paraconsistent logic - one that does not reduce to triviality in the presence of contradiction.

Belnap considers that for a basic proposition $P$, a computer might be told that $P$ is true only, false only, both true and false, or not told anything regarding $P$. The truth values of complex formulas are based on the truth values of their subformulas in a straightforward, reasonable way. Here are the truth tables for the basic connectives. They are due to Smiley, and can be found in [13]. We will refer to them later, during our proof of $\mathrm{E}$-completeness.

\begin{tabular}{c|cccc}
$\alpha \wedge \beta$ & $\mathrm{t}$ & $\mathrm{b}$ & $\mathrm{n}$ & $\mathrm{f}$ \\
\hline $\mathrm{t}$ & $\mathrm{t}$ & $\mathrm{b}$ & $\mathrm{n}$ & $\mathrm{f}$ \\
$\mathrm{b}$ & $\mathrm{b}$ & $\mathrm{b}$ & $\mathrm{f}$ & $\mathrm{f}$ \\
$\mathrm{n}$ & $\mathrm{n}$ & $\mathrm{f}$ & $\mathrm{n}$ & $\mathrm{f}$ \\
$\mathrm{f}$ & $\mathrm{f}$ & $\mathrm{f}$ & $\mathrm{f}$ & $\mathrm{f}$
\end{tabular}

\begin{tabular}{c|cccc}
$\alpha \vee \beta$ & $\mathrm{t}$ & $\mathrm{b}$ & $\mathrm{n}$ & $\mathrm{f}$ \\
\hline $\mathrm{t}$ & $\mathrm{t}$ & $\mathrm{t}$ & $\mathrm{t}$ & $\mathrm{t}$ \\
$\mathrm{b}$ & $\mathrm{t}$ & $\mathrm{b}$ & $\mathrm{t}$ & $\mathrm{b}$ \\
$\mathrm{n}$ & $\mathrm{t}$ & $\mathrm{t}$ & $\mathrm{n}$ & $\mathrm{n}$ \\
$\mathrm{f}$ & $\mathrm{t}$ & $\mathrm{b}$ & $\mathrm{n}$ & $\mathrm{f}$
\end{tabular}

\begin{tabular}{c|c}
$\alpha$ & $\neg \alpha$ \\
\hline $\mathrm{t}$ & $\mathrm{f}$ \\
$\mathrm{b}$ & $\mathrm{b}$ \\
$\mathrm{n}$ & $\mathrm{n}$ \\
$\mathrm{f}$ & $\mathrm{t}$
\end{tabular}

Starting with FDE, one can obtain a number of well known logics. Semantically this can be done by removing truth values from the truth tables of FDE. Proof theoretically, this can done by adding rules to FDE. We will specify a proof theory for FDE in Section 5.

The truth tables for the logic of paradox (LP) can be obtained from the truth tables of FDE by removing the value $n$. Designated truth values are $t$ and 
b. Here are the truth tables for LP.

\begin{tabular}{c|ccc}
$\alpha \wedge \beta$ & $\mathrm{t}$ & $\mathrm{b}$ & $\mathrm{f}$ \\
\hline $\mathrm{t}$ & $\mathrm{t}$ & $\mathrm{b}$ & $\mathrm{f}$ \\
$\mathrm{b}$ & $\mathrm{b}$ & $\mathrm{b}$ & $\mathrm{f}$ \\
$\mathrm{f}$ & $\mathrm{f}$ & $\mathrm{f}$ & $\mathrm{f}$
\end{tabular}

\begin{tabular}{c|ccc}
$\alpha \vee \beta$ & $\mathrm{t}$ & $\mathrm{b}$ & $\mathrm{f}$ \\
\hline $\mathrm{t}$ & $\mathrm{t}$ & $\mathrm{t}$ & $\mathrm{t}$ \\
$\mathrm{b}$ & $\mathrm{t}$ & $\mathrm{b}$ & $\mathrm{b}$ \\
$\mathrm{f}$ & $\mathrm{t}$ & $\mathrm{b}$ & $\mathrm{f}$
\end{tabular}

\begin{tabular}{c|c}
$\alpha$ & $\neg \alpha$ \\
\hline $\mathrm{t}$ & $\mathrm{f}$ \\
$\mathrm{b}$ & $\mathrm{b}$ \\
$\mathrm{f}$ & $\mathrm{t}$
\end{tabular}

Proof theoretically, LP can be obtained by adding to the rules of FDE the axiom scheme representing the law of excluded middle:

$$
\emptyset \vdash \phi \vee \neg \phi .
$$

Kleene's strong three-valued logic $\left(\mathrm{K}_{3}\right)$ can be obtained from FDE semantically by removing the truth value $b . t$ is the only designated truth value. Here are the truth tables for $\mathrm{K}_{3}$.

\begin{tabular}{c|ccc}
$\alpha \wedge \beta$ & $\mathrm{t}$ & $\mathrm{n}$ & $\mathrm{f}$ \\
\hline $\mathrm{t}$ & $\mathrm{t}$ & $\mathrm{n}$ & $\mathrm{f}$ \\
$\mathrm{n}$ & $\mathrm{n}$ & $\mathrm{n}$ & $\mathrm{f}$ \\
$\mathrm{f}$ & $\mathrm{f}$ & $\mathrm{f}$ & $\mathrm{f}$
\end{tabular}

\begin{tabular}{c|ccc}
$\alpha \vee \beta$ & $\mathrm{t}$ & $\mathrm{n}$ & $\mathrm{f}$ \\
\hline $\mathrm{t}$ & $\mathrm{t}$ & $\mathrm{t}$ & $\mathrm{t}$ \\
$\mathrm{n}$ & $\mathrm{t}$ & $\mathrm{n}$ & $\mathrm{n}$ \\
$\mathrm{f}$ & $\mathrm{t}$ & $\mathrm{n}$ & $\mathrm{f}$
\end{tabular}

\begin{tabular}{c|c}
$\alpha$ & $\neg \alpha$ \\
\hline $\mathrm{t}$ & $\mathrm{f}$ \\
$\mathrm{n}$ & $\mathrm{n}$ \\
$\mathrm{f}$ & $\mathrm{t}$
\end{tabular}

Proof theoretically, $\mathrm{K}_{3}$ can be obtained by adding to FDE an axiom scheme representing the principal of explosion:

$$
\phi \wedge \neg \phi \vdash \psi .
$$

Classical logic (CL) can be obtained from FDE by removing the truth values $n$ and $b . t$ is the only designated truth value. Proof theoretically, CL can be obtained by adding to FDE both the law of excluded middle and the principal of explosion.

In some sense, the motivations behind FDE and Lukasiewicz' refutation systems were similar. They sought to develop a system of logic where true/false, accept/reject are treated in equal standing. A major difference, of course, is that for Łukasiewicz, accept and reject are total and mutually exclusive categories. In FDE, true/false are arbitrary on propositional atoms.

For an introduction to FDE, see [13]. For an overview of paraconsistent logics in general, see [14].

\section{Hybrid systems, definitions and basic results}

\subsection{Definitions}

Now we will define the technical machinery and terminology needed for our hybrid deduction-refutation systems. We work over a fixed language L, with associated entailment relation $\models_{\mathrm{L}}$.

A sequent is an object of the form $\Gamma \vdash \alpha$ or $\Gamma \dashv \alpha$, where $\Gamma \cup\{\alpha\} \subset \operatorname{Form}(\mathrm{L})$, $\Gamma$ is finite, $\vdash \notin \mathrm{L}$, and $\dashv \notin \mathrm{L}$. A sequent of the first kind is called a deduction sequent. A sequent of the second kind is called a refutation sequent. 
A hybrid rule instance (or just rule instance) is a pair $\langle P, C\rangle$, where $P$ is a (possibly empty) sequence of sequents, and $C$ is a single sequent. The elements of $P$ are called the premises of the rule instance, and the sequent $C$ is called the conclusion of the rule instance. If the conclusion is a deduction sequent, the rule instance is said to be a deduction rule instance. If the conclusion is a refutation sequent, the rule instance is said to be a refutation rule instance. A set of hybrid rule instances is called a hybrid rule (or just rule).

A hybrid rule which contains only empty premises is called an axiom. If the conclusions are all deduction sequents, then it is a deduction axiom. If the conclusions are all refutation sequents, then it is a refutation axiom.

We typically represent a rule by giving one of its rule instances, and when necessary giving closure conditions. We do this in the following graphical way:

$$
\frac{\Gamma_{1} \vdash \phi_{1}, \ldots, \Gamma_{m} \vdash \phi_{m}, \Delta_{1} \dashv \psi_{1}, \ldots, \Delta_{n} \dashv \psi_{n}}{\Omega \vdash \alpha}
$$

or

$$
\frac{\Gamma_{1} \vdash \phi_{1}, \ldots, \Gamma_{m} \vdash \phi_{m}, \Delta_{1} \dashv \psi_{1}, \ldots, \Delta_{n} \dashv \psi_{n}}{\Omega \dashv \alpha}(2) .
$$

When there is no danger in doing so, we will overload the term "rule" to mean both "rule" and "rule instance." Therefore, the above will also be called simply rules, (1) is a deductive rule, and (2) is a refutation rule.

The typical closure condition for rules is uniform substitution. When rules are closed under uniform substitution, they are called structural. In particular, we typically have non-structural refutation axiom schemes, for example $p \dashv q$, where $p \neq q$ are literals. Such a rule ought not be closed under substitution, lest we erroneously derive $p \dashv p$. In this document, the refutation axiom schemes will be nonstructural, all other rules will be structural.

A deductive rule of the form of (1) above is said to be sound with respect to $L$ iff $\Omega \models_{\mathrm{L}} \alpha$ whenever

$$
\Gamma_{1} \models_{\mathrm{L}} \phi_{1}, \ldots, \Gamma_{m} \models_{\mathrm{L}} \phi_{m} \text { and } \Delta_{1} \nvdash_{\mathrm{L}} \psi_{1}, \ldots, \Delta_{n} \not \models_{\mathrm{L}} \psi_{n} .
$$

A refutation rule of the form of (2) above is said to be sound with respect to $L$ iff $\Omega \not \not_{\mathrm{L}} \alpha$ whenever

$$
\Gamma_{1} \models_{\mathrm{L}} \phi_{1}, \ldots, \Gamma_{m} \models_{\mathrm{L}} \phi_{m} \text { and } \Delta_{1} \nvdash_{\mathrm{L}} \psi_{1}, \ldots, \Delta_{n} \not \nvdash_{\mathrm{L}} \psi_{n}
$$

A hybrid derivation system is a nonempty set of hybrid rules. Note a hybrid derivation system need not contain any refutation sequents or rules. A purely deductive system is a hybrid derivation system that contains no refutation sequents in any of its rules. A purely refutational system is a hybrid derivation system that contains no deduction sequents in any of its rules.

A hybrid derivation with respect to a hybrid derivation system $\mathcal{D}$ is a finite sequence of sequents $\left\langle X_{1}, \ldots, X_{n-1}, X_{n}>\right.$, where for all $i \leq n, X_{i}$ is an instance of an axiom in $\mathcal{D}$, or else there exist $i_{1}<i, \ldots, i_{j}<i$ such that $<<X_{i_{1}}, \ldots, X_{i_{j}}>, X_{i}>$ is an instance of a rule in $\mathcal{D}$. If $<X_{1}, \ldots, X_{n-1}, X_{n}>$ 
is a hybrid derivation with $X_{n}$ as the final member of the sequence, then we say it is a hybrid derivation of $X_{n}$, and that $X_{n}$ is its conclusion.

We say $\Gamma \vdash \theta,(\Gamma \dashv \theta)$ is derivable in $\mathcal{D}$ iff there exists a hybrid derivation with respect to $\mathcal{D}$ of $\Gamma \vdash \theta,(\Gamma \dashv \theta)$. We may use $\vdash_{\mathcal{D}}$ to denote provability of sequents in $\mathcal{D}$. That is, we may write $\Vdash_{\mathcal{D}} \Gamma \vdash \theta$ or $\vdash_{\mathcal{D}} \Gamma \dashv \theta$. This is often useful in contexts where there may be confusion as to whether $\Gamma \vdash \theta$ is to be understood as a sequent - i.e. a construction proved in a hybrid system, or as a metalogical assertion that in $\mathrm{L}, \theta$ deductively follows from assumptions in $\Gamma$.

When $\mathcal{D}$ is understood, and there is no danger in doing so, however, we will typically write just $\Gamma \vdash \theta$ and $\Gamma \dashv \theta$ instead of $\Vdash_{\mathcal{D}} \Gamma \vdash \theta$ and $\vdash_{\mathcal{D}} \Gamma \dashv \theta$.

\subsection{Types of soundness and completeness}

Now that we have $\vdash$ and $\dashv$, there are more concepts available besides traditional "soundness" and "completeness." Below we give some definitions. The definitions depend on sets of formulas $\Gamma$ and formulas $\theta$ which are in the language $\mathrm{L}$. For each definition, we should quantify over all such $\Gamma$ and $\theta$. But to save space, we only write that explicitly for the first line. Assume the same quantification style for the other relevant lines.

Definitions: Given a language $\mathrm{L}$ with entailment relation $\models_{\mathrm{L}}$ and given a hybrid system $\mathcal{D}, \mathcal{D}$ is :

- deductively sound for $L$ (D-sound) iff: $\forall \Gamma \subset F \operatorname{Form}(L), \forall \theta \in F$ orm $(L)[$ $\left.\Vdash_{\mathcal{D}} \Gamma \vdash \theta \Rightarrow \Gamma \models_{\mathrm{L}} \theta\right]$,

- refutationally sound for $L$ (R-sound) iff: $\quad \Vdash_{\mathcal{D}} \Gamma \dashv \theta \Rightarrow \Gamma \nvdash_{\mathrm{L}} \theta$,

- deductively complete for $L$ (D-complete) iff: $\quad \Gamma \models_{\mathrm{L}} \theta \Rightarrow \Vdash_{\mathcal{D}} \Gamma \vdash \theta$,

- refutationally complete for $L$ (R-complete) iff: $\quad \Gamma \nvdash_{\mathrm{L}} \theta \Rightarrow \Vdash_{\mathcal{D}} \Gamma \dashv \theta$,

- Lukasiewicz sound for L (L-sound) iff it is D-sound and R-sound,

- Eukasiewicz complete for L ( -complete) iff it is D-complete and R-complete.

We also may add the qualifer "finitely" to any of these categorizations, by requiring that the condition holds only for all finite $\Gamma$. For example, $\mathcal{D}$ is finitely refutationally complete iff, for all $\Gamma \subset L$, if $\Gamma$ is finite then $\left(\Gamma \nvdash_{\mathrm{L}} \theta \Rightarrow \Vdash_{\mathcal{D}} \Gamma \dashv \theta\right)$.

\subsection{Derivative rules}

The following important definitions and propositions are given and proved in [7].

Given a deductive rule $R$, we may form derivative rules in the following way: swap places between the conclusion and one of the premises, and then "flip" the direction of the turnstile in both of those sequents.

More formally, given a deduction rule: 


$$
\frac{\Gamma_{1} \vdash \phi_{1}, \ldots, \Gamma_{m} \vdash \phi_{m}, \Delta_{1} \dashv \psi_{1}, \ldots, \Delta_{n} \dashv \psi_{n}}{\Omega \vdash \alpha}
$$

we may define, for $i \leq m$, derivative rules of the form

$$
\frac{\Gamma_{1} \vdash \phi_{1}, \ldots, \Gamma_{i-1} \vdash \phi_{i-1}, \Omega \dashv \alpha, \Gamma_{i+1} \vdash \phi_{i+1} \ldots \Gamma_{m} \vdash \phi_{m}, \Delta_{1} \dashv \psi_{1}, \ldots, \Delta_{n} \dashv \psi_{n}}{\Gamma_{i} \dashv \phi_{i}}
$$

and for $i \leq n$, derivative rules of the form

$$
\frac{\Gamma_{1} \vdash \phi_{1}, \ldots, \Gamma_{m} \vdash \phi_{m}, \Delta_{1} \dashv \psi_{1}, \ldots, \Delta_{i-1} \dashv \psi_{i-1}, \Omega \dashv \alpha, \Delta_{i+1} \dashv \psi_{i+1}, \ldots, \Delta_{n} \dashv \psi_{n}}{\Delta_{i} \vdash \psi_{i} .}
$$

The situation corresponding to derivative rules of refutation rules is analogous, so we omit it here.

Note, derivative rules as defined above require a nonempty set of premises in the original rules. Therefore, derivative rules of axioms are not defined. In the following, if we "take the derivative rules of a given rule $R$," it may be assumed that $R$ is not an axiom. If we "take the derivative rules of a set of rules $\mathcal{D}$," we mean to take the derivative rules of all rules in $\mathcal{D}$ which are not axioms.

Theorem 4.1. Let $L$ be a logical system, and $R$ a hybrid rule in the language of $L$ which is sound for $L$, then every derivative rule of $R$ is sound for $L$.

Proof. We consider the case for a deduction rule. The situation is analogous for a refutation rule. Suppose the following rule is sound for L.

$$
\frac{\Gamma_{1} \vdash \phi_{1}, \ldots, \Gamma_{m} \vdash \phi_{m}, \Delta_{1} \dashv \psi_{1}, \ldots, \Delta_{n} \dashv \psi_{n}}{\Omega \vdash \alpha}
$$

Then by the definition of soundness of a hybrid rule,

$$
\left(\Gamma_{1} \models_{\mathrm{L}} \phi_{1}, \ldots, \Gamma_{m} \models_{\mathrm{L}} \phi_{m} \text { and } \Delta_{1} \nvdash_{\mathrm{L}} \psi_{1}, \ldots, \Delta_{n} \nvdash_{\mathrm{L}} \psi_{n}\right) \Rightarrow \Omega \models \alpha .
$$

Applying some classical logic in the meta-theory, we get

$$
\begin{gathered}
\left(\Gamma_{1} \models_{\mathrm{L}} \phi_{1}, \ldots, \Gamma_{i-1} \models_{\mathrm{L}} \phi_{i-1}, \Omega \not \models_{\mathrm{L}} \alpha, \Gamma_{i+1} \models_{\mathrm{L}} \phi_{i+1} \Gamma_{m} \models_{\mathrm{L}} \phi_{m}\right. \\
\text { and } \left.\Delta_{1} \not \models_{\mathrm{L}} \psi_{1}, \ldots, \Delta_{n} \not \models_{\mathrm{L}} \psi_{n}\right) \\
\Rightarrow \Gamma_{k} \not \models \phi_{k} .
\end{gathered}
$$

Thus the corresponding derivative rule is sound.

Given a hybrid deductive system $\mathcal{D}$, its canonical extension $\mathcal{H}(\mathcal{D})$ is obtained by adding to $\mathcal{D}$ all the derivative rules of $\mathcal{D}$.

Corollary 4.1.1. If $\mathcal{D}$ is $E$-sound for $L$, then $\mathcal{H}(\mathcal{D})$ is $L$-sound for $L$.

Proof. This follows from the fact that if a rule is sound, its derivative rules are sound, combined with the fact that if $\mathcal{D}$ is E-sound, then $\Vdash_{\mathcal{D}} \Gamma \vdash \phi$ implies $\Gamma \models_{\mathrm{L}} \phi, \Vdash_{\mathcal{D}} \Delta \dashv \psi$ implies $\Delta \nvdash_{\mathrm{L}} \psi$. We need to be assured that when we have a derivation involving a derivative rule, if $\Gamma \vdash \phi$ is one of its premises, then $\Gamma \models_{L} \phi$, and if $\Delta \dashv \psi$ is one of its premises, then $\Delta \nvdash_{L} \phi$. This is guaranteed if $\mathcal{D}$ is E-sound. 
Corollary 4.1.2. If $\mathcal{D}$ is $E$-sound and D-complete for $L$, then $\mathcal{H}(\mathcal{D})$ is $L$-sound and $D$-complete. If $\mathcal{D}$ is $L$-sound and $R$-complete for $L$, then $\mathcal{H}(\mathcal{D})$ is $L$-sound and R-complete.

Proof. If $\mathcal{D}$ is Ł-sound then since $\mathcal{H}(\mathcal{D})$ is also $\mathrm{E}$-sound, we have that $\mathcal{H}(\mathcal{D})$ is a conservative extension of $\mathcal{D}$ with respect to both $\vdash$ and $\dashv$, and therefore is also $\mathrm{E}$-sound. If $\mathcal{D}$ is then $\mathrm{D}$-complete, since we cannot add any deduction sequents, $\mathcal{H}(\mathcal{D})$ is also D-complete. Similarly, if $\mathcal{D}$ is L-sound and R-complete, since we cannot add any refutation sequents, $\mathcal{H}(\mathcal{D})$ is R-complete.

Corollary 4.1.3. If $\mathcal{D}$ is a purely deductive system, and $\mathcal{D}$ is $D$-sound and $D$ complete with respect to $L$, then $\mathcal{H}(\mathcal{D})$ is $L$-sound and D-complete with respect to $L$. If $\mathcal{D}$ is a purely refutational system, and $\mathcal{D}$ is $R$-sound and $R$-complete with respect to $L$, then $\mathcal{H}(\mathcal{D})$ is $L$-sound and $R$-complete with respect to $L$.

Proof. If $\mathcal{D}$ is a purely deductive system, since it has no refutation sequents, $\mathcal{D}$ is trivially $\mathrm{R}$-sound with respect to $\mathrm{L}$. Therefore, if $\mathcal{D}$ is also $\mathrm{D}$-sound, then it is Ł-sound. If $\mathcal{D}$ is also D-complete, then by Corollary 4.1.2 $\mathcal{H}(\mathcal{D})$ is $\mathrm{E}$-sound and D-complete. The case is analogous for purely refutational systems.

Lemma 4.2. If a recursive set of rules and axioms $\mathcal{D}$ is $E$-sound and $L$ complete, then the set of validities of $\mathcal{D}$ and the set of nonvalidities of $\mathcal{D}$ are both decidable.

Proof. If $\mathcal{D}$ is E-sound and E-complete, and if the set of rules and axioms of $\mathcal{D}$ is recursive, then we have a decision procedure for the determining the validities and nonvalidities of $\mathcal{D}$.

To decide if $\Gamma \models \phi$ or $\Gamma \not \models \phi$, enumerate all the proofs of $\vdash_{\mathcal{D}} \Gamma \vdash \alpha$ and $\Vdash_{\mathcal{D}} \Gamma \dashv \alpha$, varying $\alpha$ over all formulas, which we may do since $\mathcal{D}$ is recursive. In the enumeration sequence alternate between proofs of deduction sequents and refutation sequents. Due to D-soundness and D-completeness of $\mathcal{D}, \Gamma \models \phi$ if and only if $\vdash_{\mathcal{D}} \Gamma \vdash \phi$. Due to R-soundness R-completeness of $\mathcal{D}, \Gamma \not \models \phi$ if and only if $\vdash_{\mathcal{D}} \Gamma \dashv \phi$. If $\Gamma \models \phi$, the algorithm will eventually produce $\Vdash_{\mathcal{D}} \Gamma \vdash \phi$ in the enumeration. If $\Gamma \not \models \phi$, then $\vdash_{\mathcal{D}} \Gamma \dashv \phi$ will appear in the enumeration. Either way, we have a procedure to decide if $\Gamma \models \phi$ or $\Gamma \not \models \phi$.

Corollary 4.2.1. If the set of validities of $\mathcal{D}$ is not decidable, or the set of nonvalidities of $\mathcal{D}$ is not decidable, then there is no $\mathrm{L}$-sound and $\mathrm{L}$-ccomplete extension of $\mathcal{D}$.

As noted in Goranko's paper, $\mathcal{H}(\mathcal{D})$ is too weak to be R-complete if $\mathcal{D}$ is a purely deductive system. This is because, having no refutation axioms, $\mathcal{H}(\mathcal{D})$ will not prove any refutations.

A more general question is: given a hybrid system $\mathcal{D}$, is there an algorithm by which we may establish an $\mathrm{E}$-sound and Ł-complete hybrid system $\mathcal{E}$ which is an extension of $\mathcal{D}$, for those logics $\mathcal{D}$ which admit an Ł-sound and Ł-complete extension? Would the canonical extension play a role here? 


\section{$5 \quad$ Hybrid deduction systems for four FDE based logics}

\subsection{Defining the hybrid deductive systems}

The goal of this paper is to work out a uniform treatment for FDE based systems, so let us specify a natural deduction system which is D-sound and D-complete with respect to FDE. The following is due to Font, taken from [13]. We will call the following deductive system $\mathrm{FDE}_{\mathrm{D}}$.

$$
\begin{aligned}
& A \vdash A \text { (Reflexivity Axiom) } \\
& \left.\frac{\Gamma \vdash A}{\Gamma, B \vdash A} \text { (Monotonicity (Mon) }\right) \quad \frac{\Gamma \vdash A \quad \Gamma, A \vdash B}{\Gamma \vdash B} \text { (Cut) } \\
& \frac{\Gamma, A, B \vdash C}{\Gamma A \wedge B \vdash C}(\wedge \vdash) \quad \frac{\Gamma \vdash A \quad \Gamma \vdash B}{\Gamma \vdash A \wedge B}(\vdash \wedge) \\
& \frac{\Gamma, A \vdash C \quad \Gamma, B \vdash C}{\Gamma, A \vee B \vdash C}(\vee \vdash) \\
& \frac{\Gamma \vdash A}{\Gamma \vdash A \vee B}(\vdash \vee) \quad \frac{\Gamma \vdash B}{\Gamma \vdash A \vee B}(\vdash \vee) \\
& \frac{\Gamma, A \vdash B}{\Gamma, \neg \neg A \vdash B}(\neg \neg \vdash) \quad \frac{\Gamma, \neg \neg A \vdash B}{\Gamma, A \vdash B}(\vdash \neg \neg) \\
& \frac{A \vdash B}{\neg B \vdash \neg A}(\neg) \text {. }
\end{aligned}
$$

For LP, $\mathrm{K}_{3}$, and CL we will need available the following axioms.

$\Gamma \vdash A \vee \neg A \quad($ Excluded Middle (EM)) $\quad A \wedge \neg A \vdash B \quad($ Explosion (Exp))

Let $\mathrm{LP}_{\mathrm{D}}=\mathrm{FDE}_{\mathrm{D}}+\mathrm{EM}, \mathrm{K}_{3 \mathrm{D}}=\mathrm{FDE}_{\mathrm{D}}+\operatorname{Exp}, \mathrm{CL}_{\mathrm{D}}=\mathrm{FDE}_{\mathrm{D}}+\mathrm{EM}+\operatorname{Exp}$.

Lemma 5.1. $L P_{D}$ is D-sound and D-complete with respect to $L P$. $K_{3 D}$ is $D$ sound and D-complete with respect to $K_{3} . C L_{D}$ is D-sound and D-complete with respect to $C L$.

Proof. See, for example, [14].

Now let us take the canonical extensions of our logics. Note that since $\mathrm{FDE}_{\mathrm{D}}$ differs from the other three logics only by inclusion of various axioms, and since derivative rules are not defined on axioms, all four will have the same set of derivative rules. Each rule with one premise will produce one derivative rule. Each rule with two premises will produce two derivative rules. Here are the derivative rules. 


$$
\begin{aligned}
& \frac{\Gamma, B \dashv A}{\Gamma \dashv A} \text { (antimonotonicity (antimon)) } \\
& \frac{\Gamma \dashv B \quad \Gamma, A \vdash B}{\Gamma \dashv A}\left(\text { anticut }_{1}\right) \quad \frac{\Gamma \dashv B \quad \Gamma \vdash A}{\Gamma, A \dashv B}\left(\text { anticut }_{2}\right) \\
& \frac{\Gamma, A \wedge B \dashv C}{\Gamma, A, B \dashv C}(\wedge \dashv) \\
& \frac{\Gamma \dashv A \wedge B \quad \Gamma \vdash B}{\Gamma \dashv A}(\dashv \wedge) \quad \frac{\Gamma \dashv A \wedge B \quad \Gamma \vdash A}{\Gamma \dashv B}(\dashv \wedge) \\
& \frac{\Gamma, A \vee B \dashv C \quad \Gamma, B \vdash C}{\Gamma, A \dashv C}(\vee \dashv) \quad \frac{\Gamma, A \vee B \dashv C \quad \Gamma, A \vdash C}{\Gamma, B \dashv C}(\vee \dashv) \\
& \frac{\Gamma \dashv A \vee B}{\Gamma \dashv A}(\dashv \vee) \quad \frac{\Gamma \dashv A \vee B}{\Gamma \dashv B}(\dashv \vee) \\
& \frac{\Gamma, \neg \neg A \dashv B}{\Gamma, A \dashv B}(\neg \neg \dashv) \quad \frac{\Gamma, A \dashv B}{\Gamma, \neg \neg A \dashv B}(\dashv \neg \neg) \\
& \frac{A \dashv B}{\neg B \dashv \neg A}(\operatorname{anti} \neg)
\end{aligned}
$$

It is possible some of these rules are redundant. Finding the most economical expression for an $\mathrm{L}$-sound and $\mathrm{L}$-complete hybrid deduction system is not the goal here.

In order to capture FDE, LP, $\mathrm{K}_{3}$, CL in one uniform treatment, we introduce a special shorthand. Let $\Gamma=\left\{\gamma_{0}, \ldots, \gamma_{n-1}\right\}, \Delta=\left\{\delta_{0}, \ldots, \delta_{m-1}\right\}$ be finite sets of formulas. $\Gamma$ may be empty, but $\Delta$ is nonempty. Let $\bowtie \in\{\vdash, \dashv\}$. Then

$$
\Gamma \bowtie \Delta:=\left\{\gamma_{0} \wedge \cdots \wedge \gamma_{n-1}\right\} \bowtie\left(\delta_{0} \vee \cdots \vee \delta_{m-1}\right) .
$$

Essentially, take the conjunction of the formulas on the left, and the disjunction of the formulas on the right. This is one reason why we require $\Gamma, \Delta$ to be finite. Note, $\Gamma \bowtie \Delta$ is a sequent.

We are going to specify conditions for $\Gamma \dashv \Delta$ which will count as our nonstructural refutation axiom schemes. In the left column we give the name and in the right column we give the condition on $\Gamma$ and $\Delta$.

\begin{tabular}{c|c} 
Property & Condition \\
\hline literals & For all $\psi \in \Gamma \cup \Delta, \psi$ is a literal. \\
\hline disjoint & $\Gamma \cap \Delta=\emptyset$ \\
\hline noncomplementary antecedent & For no $i \neq j \leq n$ does $\gamma_{i}=\neg \gamma_{j}$. \\
\hline noncomplementary consequent & For no $i \neq j \leq m$ does $\delta_{i}=\neg \delta_{j}$.
\end{tabular}


Here are the refutation axiom schemes (RefAx) for the logics.

$$
\begin{array}{r}
\operatorname{RefAx}(\mathrm{FDE})=\{\Gamma \dashv \Delta \mid \Gamma, \Delta \text { are finite sets of formulas that satisfy the } \\
\text { literals and disjoint properties. }\} \\
\operatorname{RefAx}(\mathrm{LP})=\{\Gamma \dashv \Delta \mid \Gamma, \Delta \text { are finite sets of formulas that satisfy the } \\
\text { literals, disjoint, and } \\
\text { noncomplementary consequent properties. }\} \\
\operatorname{RefAx}\left(\mathrm{K}_{3}\right)=\{\Gamma \dashv \Delta \mid
\end{array}
$$

Theorem 5.2. For $L \in\left\{F D E, L P, K_{3}, C L\right\}$, Ref $A x(L)$ is $R$-sound with respect to $L$.

Proof. Let L be a logic among FDE, LP, K 3 , CL. Let $\Gamma \dashv \Delta$ be an instance of $\operatorname{RefAx}(\mathrm{L})$. We wish to show $\Gamma \nvdash_{\mathrm{L}} \Delta$. We will argue by cases for each logic. For each L we demonstrate a model $*$ which satisfies $\Gamma$ and does not satisfy $\Delta$.

- Case: $\mathrm{L}=\mathrm{FDE}$ :

- Let $\gamma^{*}=b$ for all $\gamma \in \Gamma$ and let $\delta^{*}=n$ for all $\delta \in \Delta$. This is possible to do since $\Gamma, \Delta$ are disjoint sets of literals.

- Case $\mathrm{L}=\mathrm{LP}$ :

- Let $\gamma^{*}=b$ for all $\gamma \in \Gamma$. If $q=\delta \in \Delta$ for some propositional variable $q$, then let $q^{*}=f$. If $\delta=\neg q$, let $q^{*}=t$. This is possible to do since $\Gamma, \Delta$ are disjoint sets of literals, and because $\Delta$ contains no complementary literals.

- Case $\mathrm{L}=\mathrm{K}_{3}$ :

- Let $\delta^{*}=n$ for all $\delta \in \Delta$. If $p=\gamma \in \Gamma$ for some propositional variable $p$, then let $p^{*}=t$. If $\gamma=\neg p$, let $p^{*}=f$. This is possible to do since $\Gamma, \Delta$ are disjoint sets of literals, and because $\Gamma$ contains no complementary literals.

- Case $\mathrm{L}=\mathrm{CL}$ :

Australasian Journal of Logic (18:6) 2021, Article no. 1 
- If $p=\gamma \in \Gamma$ for some propositional variable $p$, then let $p^{*}=t$. If $\gamma=\neg p$, let $p^{*}=f$. If $q=\delta \in \Delta$ for some propositional variable $q$, then let $q^{*}=f$. If $\delta=\neg q$, let $q^{*}=t$. This is possible to do since $\Gamma, \Delta$ are disjoint sets of literals, and neither $\Gamma$ nor $\Delta$ contains complementary literals.

We now define the hybrid derivation systems for our logics. In the next section we will prove their $\mathrm{E}$-soundness and $\mathrm{E}$-completeness for finite antecedent.

- $\mathrm{FDE}_{\mathrm{H}}=\mathcal{H}\left(\mathrm{FDE}_{\mathrm{D}}\right)+\operatorname{RefAx}(\mathrm{FDE})$.

- $\mathrm{LP}_{\mathrm{H}}=\mathcal{H}\left(\mathrm{LP}_{\mathrm{D}}\right)+\operatorname{RefAx}(\mathrm{LP})$.

- $\mathrm{K}_{3 \mathrm{H}}=\mathcal{H}\left(\mathrm{K}_{3 \mathrm{D}}\right)+\operatorname{RefAx}\left(\mathrm{K}_{3}\right)$.

- $\mathrm{CL}_{\mathrm{H}}=\mathcal{H}\left(\mathrm{CL}_{\mathrm{D}}\right)+\operatorname{RefAx}(\mathrm{CL})$.

In [7], Goranko also defined a hybrid derivation system which is Ł-sound and complete for classical logic. He took the canonical extension of a cut-free formulation for classical logic to produce his system. The advantages of his system are that it is cut-free, and it does not rely on the anticut rules. One advantage of our system is that though it does use cut and anticut, it is fairly general. One proof technique will prove $\mathrm{E}$-soundness and completness for all four logics involved. Due to the uniformity of our approach, the major differences between the calculi appear at the level of their axiom and refutation axiom schemes.

\section{2 Ł-soundness and Ł-completeness}

Theorem 5.3. For $L \in\left\{F D E, L P, K_{3}, C L\right\}, L_{H}$ is $E$-sound and finitely $L$ complete with respect to $L$.

Proof. Let L be among FDE, KP, $\mathrm{K}_{3}, \mathrm{CL}$. We will show that $\mathrm{L}_{\mathrm{H}} \mathrm{L}$-sound and finitely $\mathrm{E}$-complete. It is enough to show that $\mathrm{L}_{\mathrm{H}}$ is $\mathrm{D}$-sound, $\mathrm{D}$-complete, R-sound, and finitely R-complete.

To see that $\mathrm{L}_{\mathrm{H}}$ is $\mathrm{D}$-complete, note that $\mathrm{L}_{\mathrm{H}}$ is an extension of $\mathrm{L}_{\mathrm{D}}$, which is D-complete. Therefore, since $\mathrm{L}_{\mathrm{D}}$ proves all valid entailments, so too does $\mathrm{L}_{\mathrm{H}}$. Thus $\mathrm{L}_{\mathrm{H}}$ is D-complete.

To see that $\mathrm{L}_{\mathrm{H}}$ is $\mathrm{D}$-sound, note that if a rule of $\mathrm{L}_{\mathrm{H}}$ has as its conclusion a deduction sequent, then that rule was already in $L_{D}$. Therefore, since $L_{D}$ is D-sound, so is $\mathrm{L}_{\mathrm{H}}$.

To see that $\mathrm{L}_{\mathrm{H}}$ is $\mathrm{R}$-sound, note that if a rule of $\mathrm{L}_{\mathrm{H}}$ has as its conclusion a refutation sequent, then that rule is either a refutation axiom of $\operatorname{Ref} A x(L)$, or it is a derivative rule of some rule in $\mathrm{L}_{\mathrm{D}}$. In the former case, we have shown that $\operatorname{RefAx}(\mathrm{L})$ is R-sound with respect to $\mathrm{L}$ (Theorem 5.2). In the latter case, since $\mathrm{L}_{\mathrm{D}}$ is a purely deductive system, then by Corollary 4.1 .3 the set of derivative 
rules of $L_{D}$ is $R$-sound with respect to $L$. In either case, if $\vdash_{L_{H}} \Gamma \dashv \alpha$ then $\Gamma \not{ }_{L} \alpha$, so $\mathrm{L}_{\mathrm{H}}$ is R-sound.

We will now prove R-completeness for finite $\Gamma$.

Suppose for some finite $\Gamma$ that $\Gamma \nvdash_{L} \alpha$. We wish to show $\Gamma \dashv_{L} \alpha$. We will omit the L subscript on $\dashv$ moving forward. This is an adaptation of Goranko's proof in [7]. His proof did not use anticut. Our's does, and thus allows us to prove the same results, without the intermediary lemmas he uses. The problem is, his lemmas are not true for some of our logics, so we cannot follow his proof directly.

First assume $\Gamma$ and $\alpha$ are in conjunctive normal form. That is

$$
\Gamma=\bigwedge_{i=1}^{m} A^{i} \quad A^{i}=\bigvee_{j=1}^{m_{i}} B_{j}^{i} \quad \alpha=\bigwedge_{k=1}^{n} C^{k} \quad C^{k}=\bigvee_{l=1}^{n_{k}} D_{l}^{k}
$$

where $B_{j}^{i}$ and $D_{l}^{k}$ are all literals.

Let $D(\mathrm{~L})$ be the set of designated values of the logic $\mathrm{L}$. Since $\Gamma \not \models \alpha$, there is a model $*$ such that for all $\gamma \in \Gamma, \gamma^{*} \in D(\mathrm{~L})$, yet $\alpha^{*} \notin D(\mathrm{~L})$. Fix one such model $*$.

For a literal $p$, define $p^{\circ}$ as $p^{\circ}=p$ if $p^{*} \in D(\mathrm{~L})$, and $p^{\circ}=\neg p$ if $p^{*} \notin D(\mathrm{~L})$. For a set of formulas $X$, define $\operatorname{Lit}(X)=\{p \mid p$ is a literal in $X\}$. Then define $\Gamma^{\circ}=\left\{p^{\circ} \mid p \in \operatorname{Lit}(\Gamma)\right\}$. It is clear that $\Gamma^{\circ}$ satisfies $*$, therefore $\Gamma^{\circ} \nvdash_{\mathrm{L}} \alpha$. Then $\Gamma^{\circ} \nvdash_{\mathrm{L}} C^{k}$ for some $k \leq n$. That is, $\Gamma^{\circ} \forall_{\mathrm{L}} \bigvee_{l=1}^{n_{k}} D_{l}^{k}$. Since $D_{1}^{k}, \ldots, D_{n_{k}}^{k}$ are all literals, if we let $\Delta_{k}=\operatorname{Lit}\left(\left\{\bigvee_{l=1}^{n_{k}} D_{l}^{k}\right\}\right)$ we have $\Gamma^{\circ} \cap \Delta_{k}=\emptyset$.

Depending on our logic, we may have some more conditions on $\Gamma^{\circ}$ and $\Delta_{k}$. Since $\Gamma^{\circ}$ is satisfiable, this means that if we are working in $\mathrm{CL}$ or $\mathrm{K}_{3}, \Gamma^{\circ}$ contains no complimentary literals. Since $\bigvee_{k=1}^{n_{i}} D_{l}^{k}$ is falsifiable, this means that if we are working in CL or LP, $\Delta_{k}$ contains no complimentary literals. We see that in all four cases, $\Gamma^{\circ} \dashv \Delta_{k}$ is an instance of a refutation axiom for L.

We pause this train of thought for a moment and turn to $\Gamma$. We want to show $\Gamma^{\circ} \vdash \Gamma$. Since $\Gamma$ is satisfied by $*, A^{i}$ is satisfied by $*$ for each $i \leq m$. Then, for each $i$, there exists a $r_{i} \leq m_{i}$ such that $B_{r_{i}}^{i}$ is satisfied by $*$. Thus $\left(B_{r_{i}}^{i}\right)^{\circ}=B_{r_{i}}^{i}$, so $B_{r_{i}}^{i} \in \Gamma^{\circ}$. Thus for each $i \leq m, \Gamma^{\circ} \vdash B_{r_{i}}^{i}$, so $\Gamma^{\circ} \vdash A^{i}$. Applying some standard FDE logic, we get $\Gamma^{\circ} \vdash \Gamma$.

Here, now, is the proof of $\Gamma \dashv \alpha$. (Some lines in the proof merely rename terms in the preceding line. They are included only to aid readability of the proof.)

$$
\begin{aligned}
& \frac{\Gamma^{\circ} \dashv \Delta_{k} \quad(\operatorname{RefAx}(\mathrm{L}))}{\frac{\Gamma^{\circ} \dashv \bigvee_{l=1}^{n_{k}} D_{l}^{k}}{\Gamma^{\circ} \dashv C^{k}}} \\
& \frac{\Gamma^{\circ \dashv C^{k}} \Gamma^{\circ}, \bigwedge_{j=1}^{n} C^{j} \vdash C^{k}}{\frac{\Gamma^{\circ} \dashv \bigwedge_{j=1}^{n} C^{j}}{\frac{\Gamma^{\circ} \dashv \alpha}{\text { anticut }_{1}}}} \frac{\Gamma^{\circ} \vdash \Gamma}{\frac{\Gamma^{\circ}, \Gamma \dashv \alpha}{\Gamma \dashv \alpha} \text { (antimon) }}\left(\text { anticut }_{2}\right)
\end{aligned}
$$


Now if $\Gamma$ is not a single formula in normal form, or if $\alpha$ is not in normal form, we will show the result still holds.

Let $\Gamma=\left\{\gamma_{1}, \ldots, \gamma_{n}\right\}$ be a finite set of formulas. Let $\tilde{\Gamma}$ be the conjunctive normal form of the conjunction of all formulas in $\Gamma$. Let $\tilde{\alpha}$ be the conjunctive normal form of $\alpha$. We have the following proof of $\Gamma \dashv \alpha$, given $\tilde{\Gamma} \dashv \tilde{\alpha}$.

$$
\begin{aligned}
& \frac{\tilde{\Gamma} \dashv \tilde{\alpha} \quad \tilde{\Gamma} \vdash \gamma_{1}}{\tilde{\Gamma}, \gamma_{1} \dashv \tilde{\alpha}}\left(\text { anticut }_{2}\right)
\end{aligned}
$$

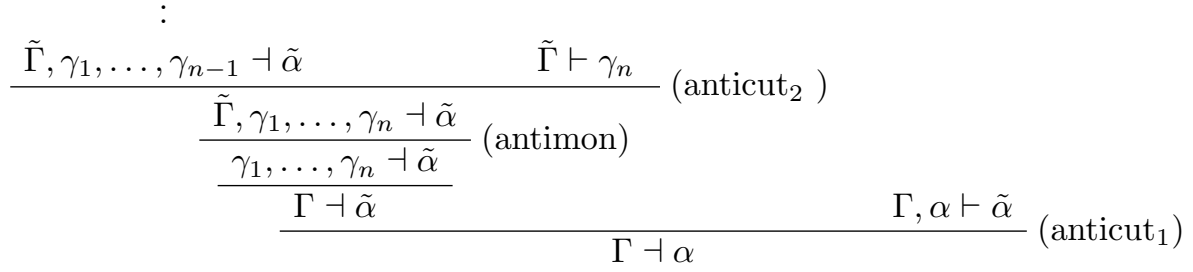

\subsection{Admissibility of rules}

Given a hybrid derivation system $\mathcal{D}$, a rule $R$ is admissible with respect to $\mathcal{D}$ if no new sequents are proved in the expanded system $\mathcal{D} \cup\{R\}$. A set of rules $\mathcal{E} \subset \mathcal{D}$ is redundant in $\mathcal{D}$ if each rule in $\mathcal{E}$ is admissible in $\mathcal{D} \backslash \mathcal{E}$.

Corollary 5.3.1. The set of rules $\{\wedge \dashv, \vee \dashv, \dashv \vee, \neg \neg \dashv, \dashv \neg \neg$, anti $\neg\}$ are redundant in $F D E_{H}, L P_{H}, K_{3 H}, C L_{H}$.

Proof. In the proofs of R-completeness above, the only rules used were refutation axioms, anticut ${ }_{1}$, anticut ${ }_{2}$, antimonotonicity, and $\dashv \wedge$.

\section{$6 \quad$ Final thoughts}

Hybrid refutation deduction systems, though not new, are a relatively unexplored area of proof theory. We believe that they warrant further attention, and are of interest to the logic community for at least three reasons.

First, they are a novel kind of deductive system, and thus intrinsically worthwhile to the curious logician. It would be philosophically interesting and likely fruitful to follow Lukasiewicz and consider the consequences of treating "accept" and "reject" on par.

Second, they offer the logician a new tool, namely the ability to prove validities and nonvalidities syntactically in the same framework. While for a given logic we may already have access to countermodels to prove nonvalidity of a sequent, having a syntactic method to do so provides the logician or automated theorem prover an additional option. Moreover, for some logics constructing 
countermodels may be difficult or computationally expensive. In such cases a syntactic approach may be preferable.

Third, being able to prove both deduction and refutation sequents, hybrid systems offer the possibility of shorter proof lengths and increased computational efficiency. Though we do not touch on computational matters in this paper, future studies should seek to quantify the trade-offs and benefits of using hybrid systems for formal proof writing and proof search.

In this paper, we offer original L-sound and complete hybrid systems for four FDE-based logics, namely FDE, LP, $\mathrm{K}_{3}$, and CL. As far as we know, these are the first $\mathrm{E}$-sound and complete systems for LP and $\mathrm{K}_{3}$. The main technique used is a continuation of a key idea in [7] - to create an E-sound and complete hybrid system from the canonical extension of a purely deductive system, along with appropriate refutation axiom schemes. One main contribution of this work is to demonstrate the creation of multiple $\mathrm{L}$-sound and complete hybrid extensions at the same time, using a uniform procedure.

The generality of this technique suggests that it may have fairly wide application. Further studies should apply it to more purely deductive systems extensions of FDE would be a good place to start. We believe this work is an early step towards developing a general theory of $€$-sound and complete hybrid extensions of pure systems. The ultimate goal here would be an algorithm to create an E-sound and complete hybrid extension whenever possible.

Now if a logic is not decidable, then as discussed earlier no recursively axiomatized hybrid system will be Ł-sound and Ł-complete for it. But for those purely deductive systems which are decidable, it would be prudent to explore in greater depth their canonical extensions. A natural question to ask is, if $\mathcal{D}$ is a purely deductive system, and is D-sound and complete with respect to a logic $\models_{L}$, is there a (finite, recursively enumerable) set of refutation axioms $\operatorname{Ref} \operatorname{Ax}(\mathrm{L})$ such that $\mathcal{H}(\mathcal{D})+\operatorname{Ref} A x(\mathrm{~L})$ is $\mathrm{E}$-sound and complete with respect to $\models_{L}$ ? If yes, can we constructively produce such a set of refutation axioms? If yes again, we have essentially achieved the goal of giving a mechanical procedure to turn a D-sound and complete purely deductive system into an $\mathrm{E}$-sound and complete hybrid system. That goal in the general case may be too ambitious. Yet, perhaps there are classes of deductive systems for which it is attainable.

Towards the task of specifying refutation axioms, it is interesting to note a duality between the refutation and deduction axioms of LP and $\mathrm{K}_{3}$.

- $\Gamma \vdash \Delta$ is a deduction axiom of LP only if $\Delta$ contains a complementary pair.

- $\Gamma \dashv \Delta$ is a refutation axiom of LP only if $\Gamma$ contains no noncomplementary pairs.

- $\Gamma \vdash \Delta$ is a deduction axiom of $\mathrm{K}_{3}$ only if $\Gamma$ contains a complementary pair.

- $\Gamma \dashv \Delta$ is a refutation axiom of $K_{3}$ only if $\Delta$ contains no noncomplementary pairs. 
Does the close symmetry between deduction axioms and refutation axioms seen here give us a clue as to how to produce refutation axiom schemes for Ł-sound and complete hybrid extensions in the general case?

Finally, to be a tool with practical applications in automated theorem proving, hybrid systems should allow elimination of cut and anticut rules. We could of course obtain anitcut free formulations automatically by taking the canonical extension of a cut-free system. The anticut rules are the derivative rules of cut, so if there is no cut, there are no anticut rules in the canonical extension. This is the approach taken in [7]. However, proving Ł-soundness and completeness in such cases appears to be rather idiosyncratic to the logic at hand. Much of the uniformity of our proofs arose through liberal use of anticut, and it is not immediately clear how to eliminate anticut from our proofs. Still, future work should try to develop a uniform procedure to prove $\mathrm{E}$-soundness and completeness in such hybrid systems. Alternatively, for a given class of hybrid systems which do contain cut and anticut, develop a uniform procedure to eliminate cut and anticut in them.

This line of research opens the door to exploring the relationship between cut and anticut rules in general. For example, if $\vdash$ admits cut elimination, does $\dashv$ admit elimination of the anticut rules? Can a cut-elimination procedure be adapted to produce an anticut-elimination procedure?

\section{References}

[1] N. D. Belnap. "How a computer should think". In: Contemporary aspects of philosophy. Ed. by G. Ryle. Oriel Press, 1977, pp. 30-55. URL: https : //doi.org/10.1007/978-3-030-31136-0_4. Reprinted in: Entailment: the logic of relevance and necessity Vol. 2, by A.R. Anderson, N.D. Belnap, J.M. Dunn, Princeton, 1992, pp 506-541.

[2] P. Bonatti and A.C. Varzi. "On the meaning of complementary systems". In: 10th International Congress of Logic, Methodology, and Philosophy of Science. Volume of Abstracts. 1995, p. 122.

[3] R.T. Brady. "A rejection system for the first-degree formulae of some relevant logics". In: Australasian Journal of Logic 6 (2008), pp. 55-69. URL: https://doi.org/10.26686/ajl.v6i0.1793.

[4] R. Caferra and N. Peltier. "Accepting/rejecting propositions from accepted/rejected propositions: a unifying overview". In: International Journal of Intelligent Systems 23.10 (2008), pp. 999-1020. URL: https://doi . org/10.1002/int. 20304.

[5] A. Citkin. "A meta-logic of inference rules: syntax". In: Logic and Logical Philosophy 24 (2014), pp. 313-337. URL: https://doi.org/10.12775/ LLP. 2015.007.

[6] J. M. Dunn. "Intuitive semantics for first-degree entailment and 'coupled trees"'. In: Philosophical Studies 29.3 (1976), pp. 149-168. URL: https: //doi.org/10.1007/978-3-030-31136-0_3. 
[7] V. Goranko. "Hybrid deduction-refutation systems". In: Axioms 8.4 (2019), p. 118. URL: https://doi.org/10.3390/axioms8040118.

[8] V. Goranko. "Refutation systems in modal logic". In: Studia Logica 53.2 (1994), pp. 299-324. URL: https://doi.org/10.1007/BF01054714.

[9] V. Goranko, G. Pulcini, and T. Skura. "Refutation systems: an overview and some applications to philosophical logics". In: Knowledge, Proof and Dynamics (2020), pp. 173-197. URL: https://doi.org/10.1007/978981-15-2221-5_9.

[10] R. Goré and L. Postniece. "Combining derivations and refutations for cut-free completeness in bi-intuitionistic logic". In: Journal of Logic and Computation 20.1 (2010), pp. 233-260. URL: https://doi.org/10.1093/ logcom/exn067.

[11] J. Ł ukasiewicz. Aristotle's syllogistic from the standpoint of modern formal logic. Oxford: Clarendon Press, 1951.

[12] E.G.K López-Escobar. "Refutability and elementary number theory". In: Indagationes Mathematicae 34 (1972), pp. 362-37. URL: https://doi. org/10.1016/1385-7258(72)90053-4.

[13] H. Omori and H. Wansing. "40 years of FDE: an introductory overview". In: Studia Logica 105.6 (2017), pp. 1021-1049. URL: https://doi.org/ 10.1007/s11225-017-9748-6.

[14] G. Priest. "Paraconsistent logic". In: Handbook of Philosophical Logic. Ed. by D.M Gabbay and F. Guenthner. Vol. 6. Springer, Dordrecht, 2002, pp. 287-393. URL: https://doi.org/10.1007/978-94-017-0460-1_4.

[15] D. Scott. "Completeness proofs for the intuitionistic sentential calculus". In: Summaries of talks presented at the Summer Institute of Symbolic Logic. Cornell University, 1957, pp. 231-241.

[16] T. Skura. "Maximality and refutability". In: Notre Dame Journal of Formal Logic 45.2 (2004), pp. 65-72. URL: https://doi.org/10.1305/ ndjfl/1095386644.

[17] T. Skura. "Refutation calculi for certain intermediate propositional logics". In: Notre Dame Journal of Formal Logic 33.4 (1992), pp. 552-560. URL: https://doi.org/10.1305/ndjfl/1093634486.

[18] A Tamminga and K Tanaka. "A Natural Deduction System for First Degree Entailment". In: Notre Dame Journal of Formal Logic 40.2 (1999), pp. 258-272. URL: https://doi.org/10.1305/ndjfl/1038949541.

[19] H. Wansing. "Proofs, disproofs, and their duals". In: Advances in Modal Logic. Ed. by V. Goranko, L. Beklemishev, and V. Shehtman. Vol. 8. 2010, pp. 483-505. 